\title{
Applications of Complex-Valued Self-Organizing Maps to Ground Penetrating Radar Imaging Systems
}

\author{
Akira Hirose and Yukimasa Nakano \\ Department of Electrical Engineering and Information Systems, The University of Tokyo \\ Japan
}

\section{Introduction}

We have been studying ground penetrating radars (GPRs) to visualize antipersonnel plastic landmines by dealing with complex-amplitude (amplitude and phase) data of scattered and/or reflected electromagnetic wave by using a complex-valued self-organizing map (CSOM). In GPRs, we cannot get rid of intense clutter because of the ground surface, soil clods, stones, unexploded ordnance, and exploded ones scattered as metal fragments. We need to discriminate plastic landmines from those other objects with a high correct discrimination rate. In our systems, we pay attention to complex-amplitude texture, instead of intensity used in conventional radar systems, in the spatial and frequency domains to extract textures. Then we feed the textural feature to a CSOM so that we classify the textures adaptively, and visualize the plastic landmines. We have been inspired by the human early vision and the self-organizing adaptability. In this Chapter, we present the system construction, explain the procedure and dynamics, and demonstrate the effectiveness in experiments.

The organization of this chapter ${ }^{1}$ is as follows. In Section 2, we present the general background of plastic landmine detection. Section 3 explains the merits and demerits of three plastic landmine detection systems that utilize the electromagnetic wave. Section 4 describes the features of and merits in complex-valued neural networks including self-organizing maps. Then we present the processing flow of our plastic landmine detection system based on the CSOM and show the conventional and proposal methods in Section 5. In Section 6, we show experimental results with observed data. We summarize and conclude this chapter in Section 7.

\section{Landmine detection and ground penetrating radars (GPRs)}

GPRs are widely used for buried object detection, ruin investigation, groundwater assessment, and other various applications (Sato \& Takeshita, 2000) (Moriyama et al., 1997). They are also expected to find nonmetallic landmines (Bourgeois \& Smith, 1998)(Montoya \& Smith, 1999) (Peters Jr. \& Daniels, 1994) (Sato et al., 2004) (Sato et al., 2005)

\footnotetext{
${ }^{1}$ A part of this chapter was published in Nakano \& Hirose (2009c): Y.Nakano and A.Hirose, "Adaptive ground penetrating radar systems to visualize antipersonnel plastic landmines based on local texture in scattering and reflection data in space and frequency domains," in Radar Technology, In-Tech, (Dec. 2009) pp.127-140, ISBN 978-953-307-029-2.
} 
(Sato et al., 2006). A near future target is to find or visualize antipersonnel landmines with a high distinction rate between landmines and other objects. The conventional metal detectors, based on electromagnetic induction, use so low frequency that the electromagnetic field penetrates through the soil very deep, and the false negative probability is very small. However, because of its long wavelength, the resolution is limited, and they can tell just whether inductive material exists or not. They cannot distinguish landmines from other metal fragments. GPRs employ much higher frequency. Then the resulting higher resolution will be helpful to discriminate landmines.

Currently, there are two methods to remove the plastic landmines. One is a metal detector, and the other is a rotary cutter. The former detects a blasting cap made of metal in the landmine. Because the cap is very small, we must set the sensitivity at a high level. Then, the positive fault rate is as high as about $99.9 \%$ (specificity=0.1\%), resulting in a lot of time to remove the landmines. The latter, rotary cutter, looks like a bulldozer, bores the ground and tries to clear the landmines by exploding them. The problems in this method are necessity of additional removal by human because of impossibility of perfect clearance, necessity of sufficient areas for the approach of the rotary cutter, and land pollution by the exploded powder.

Accordingly, though these methods have certain merits, they have also demerits. Therefore, new landmine detection systems based on GPRs attract attention and are studied by a dozen of researcher groups/laboratories presently to solve the problem. Most of the proposed methods employ high-frequency and wide-band electromagnetic wave to visualize a plastic landmine itself instead of the metallic blasting cap. In general, it is noted that the electromagnetic wave is reflected at boundaries between materials having different permittivity, and that the spatial resolution of the observation is almost the same as the wavelength. Therefore, it is possible to detect not only the metal but also the plastic body because the electromagnetic wave is reflected at the boundary of the soil and the plastic. In addition, the wide band electromagnetic wave has the possibility to observe the the accurate distance from the antenna to the target, physical property for electromagnetic wave and structural characteristics of the target. That is to say, a GPR system has a potential of detecting plastic landmines more strictly than the metal detector does.

However, high-frequency wave also induce a lot of clutter, which is caused by the roughness of the earth's surface and scattering substances other than the plastic landmines. Consequently, it is very difficult to extract significant features helpful for detecting the plastic landmines from the observed data by ignoring the clutter. Furthermore, it is also difficult to treat the extracted features effectively. A lot of processing methods were proposed so far. That is, we must resolve the following two steps to detect the plastic landmines. The first step is how to extract the features, and the second is how to treat the extracted features. To accomplish our goals, we must select or develop new effective methods.

Previously we proposed an adaptive radar imaging system to visualize plastic landmines using complex-valued self-organizing map (CSOM) (Hara \& Hirose, 2004) (Hara \& Hirose, 2005). The CSOM is a type of complex-valued neural networks (Hirose, 2006) (Hirose, 2009) (Hirose, 2003) (Aizenberg et al., 2000) (Nitta, 2009) (Mandic \& Goh, 2009) (Hirose, n.d.) (Widrow et al., 1975), which will be described briefly in Section 4 . With the system, we observe reflection and scattering to obtain a complex-amplitude two-dimensional image at multiple frequencies. In the resulting 3-dimensional (2-dimensional (space) $\times$ frequency) data, we extract local texture information as a set of feature vectors, and feed them to a CSOM for adaptive classification of the 3-dimensional texture. By using the system, we could visualize antipersonnel plastic landmines buried shallowly underground. We also constructed 
a preliminary object identifier, which is a type of associative memory that learns the feature of the plastic-landmine class with adaptation ability to various ground conditions (Hirose et al., 2005) (Nakano \& Hirose, 2009a). However, the system requires a long observation time because it employs mechanical scan. Long observation time is one of the most serious problems in high-resolution imaging systems. Some methods to overcome the problem have been investigated (Kobayashi et al., 2004) (Shrestha et al., 2004).

We then developed a portable visualization system with an antenna array to reduce the observation time (Masuyama \& Hirose, 2007). The array has $12 \times 12$ antenna elements, resulting in about 144 pixels. The element aperture size is $28 \mathrm{~mm} \times 14 \mathrm{~mm}$, which determines the spatial resolution. In texture evaluation and adaptive CSOM classification, a higher resolution leads to a better performance. We recently proposed a resolution enhancement method using a special antenna-selection manner in combination with elimination of direct coupling and calibration of propagation pathlength difference (Masuyama et al., 2007) (Masuyama et al., 2008). However, even with such resolution enhancement, the visualization performance is still worse than that obtained with the first mechanical-scanning system. The resolution is still insufficient, and the mutual coupling between antenna elements are not completely ignorable.

In this chapter, we describe two techniques to improve the visualization ability without mechanical scan, namely, the utilization of SOM-space topology in the CSOM adaptive classification and a feature extraction method based on local correlation in the frequency domain. In experimental results, we find that these two techniques improve the visualization performance significantly. The local-correlation method contributes also to the reduction of tuning parameters in the CSOM classification.

\section{Conventional technology}

Roughly speaking, there are two radar systems. One is the pulse radar, and the other is the stepped-frequency radar. In the following subsections, we briefly explain the characteristics of these methods respectively.

\subsection{Pulse GPR}

A pulse GPR observes the time response of the electromagnetic-wave pulse irradiated toward the ground. The time response represents the depth of a scatterer. When we sweep an antenna in two dimension horizontally, we can obtain the three dimensional data. Besides, as the electromagnetic wave is reflected at the boundary of materials that have different permittivity, it is possible to observe the reflection wave from the plastics that forms a landmine, not only the metal blasting cap. The pulse GPRs have another merit. As the pulse have a wide bandwidth, as wide as that of the stepped-frequency range mentioned below, we can observe not only the time response but also the frequency response through the Fourier transform, and these data may show characteristics of the target. Regarding the measuring time, we can conduct the measurement more speedily in comparison with the stepped-frequency GPRs. However, as a maximum frequency component of the pulse radar is usually about $f=6 \mathrm{GHz}$ at the most. That is, the shortest free-space wavelength is about $5 \mathrm{~cm}$. Then the pulse GPRs cannot observe sufficient amount of characteristics of plastic landmines whose size is typically the same as the wavelength. Besides, the ground surface is very rough, and the soil, including various scatterers, often causes serious clutter. Therefore, it is very difficult to obtain clear images. To solve this problem we need to utilize sharper pulse which consists of a wide-band 
wave with a high power. However, as a high-peak pulse is distorted by the nonlinearity in transmitter circuits and switched antennas, the problem cannot be solved sufficiently.

\subsection{Stepped-frequency GPR}

Stepped-frequency GPRs observe the reflected continuous wave at a wide-range frequency points. This method does not need to output strong power instantaneously. Then, the electromagnetic wave has little influence on the nonlinearity of the circuits and the switches. As the results, stepped-frequency GPR accomplishes higher SN ratio than the pulse GPR. In addition, it is easier for the stepped-frequency radar to observe the high frequency wave and select bandwidth freely than a pulse radar system. Besides, we can obtain the time response, like what pulse radar provide, through the inverse Fourier transform of the observed frequency domain data.

Here we present an example of raw data obtained by a stepped-frequency radar system having a relatively high frequency range with mechanical scanning in Fig.1(a) so that the readers can catch what type of data we are dealing with. This is a rather simple example for observation of $30 \mathrm{~cm} \times 30 \mathrm{~cm}$ area of sand (rich in silica) soil. The frequency is changed stepwise from the top-left to bottom-right. The brightness shows the intensity, while the hue represents the phase. Though the complex-amplitude texture (pattern) seems almost at random, the randomness is varying place by place. In this experiment, we have a plastic landmine (TYPE-72 amtipersonnel plastic landmine) at the center of the observation area in this case. Readers may find a certain feature in the randomness at the central area. In addition, we can actually find similar changes there also in the frequency domain. The human beings are highly sensitive to such changes in the textural features. We extract the features specific to the landmine area based on the human early vision system in the complex-amplitude domain by using the CSOM to visualize the landmine area as shown at the bright area at the center in Fig. 1(b).

As above, stepped-frequency GPRs enables us to accomplish high SN ratio at the high frequency with a wide band, and obtain not only the time response but also frequency domain feature very effectively. It is true that even the stepped-frequency GPR has a drawback. Namely, it takes too long time to measure the scattering because of the time required for frequency sweeping. However, this problem will be solved by inventing new appropriate devices in the near future.

Then we can expect a higher precision with the system utilizing the stepped-frequency GPR than the conventional systems. To achieve this purpose, there are two important points we should consider carefully. One is to extract useful features from the obtained data, and the other is to fully utilize the features. However, a perfect technique has not been suggested yet. In the next section, we show the details of our CSOM-based signal processing published in our previous paper (Nakano \& Hirose, 2009b).

\section{Complex-valued neural networks including self-organizing maps}

Complex-valued neural networks are the networks that deal with complex signals with complex variables in the complex domain (Hirose, 2006) (Aizenberg et al., 2000) (Hirose, 2003) (Nitta, 2009) (Mandic \& Goh, 2009). They are also extensions of various complex-valued linear signal processing methods such as complex least mean square (LMS) algorithm (Widrow et al., 1975). The behavior of a $N$-dimensional complex-valued neural networks is different from that of $2 \mathrm{~N}$-dimensional real-valued one (Hirose, 2009). The origin lies in the nature of multiplication in the complex domain. That is, the weight multiplication at synapses 


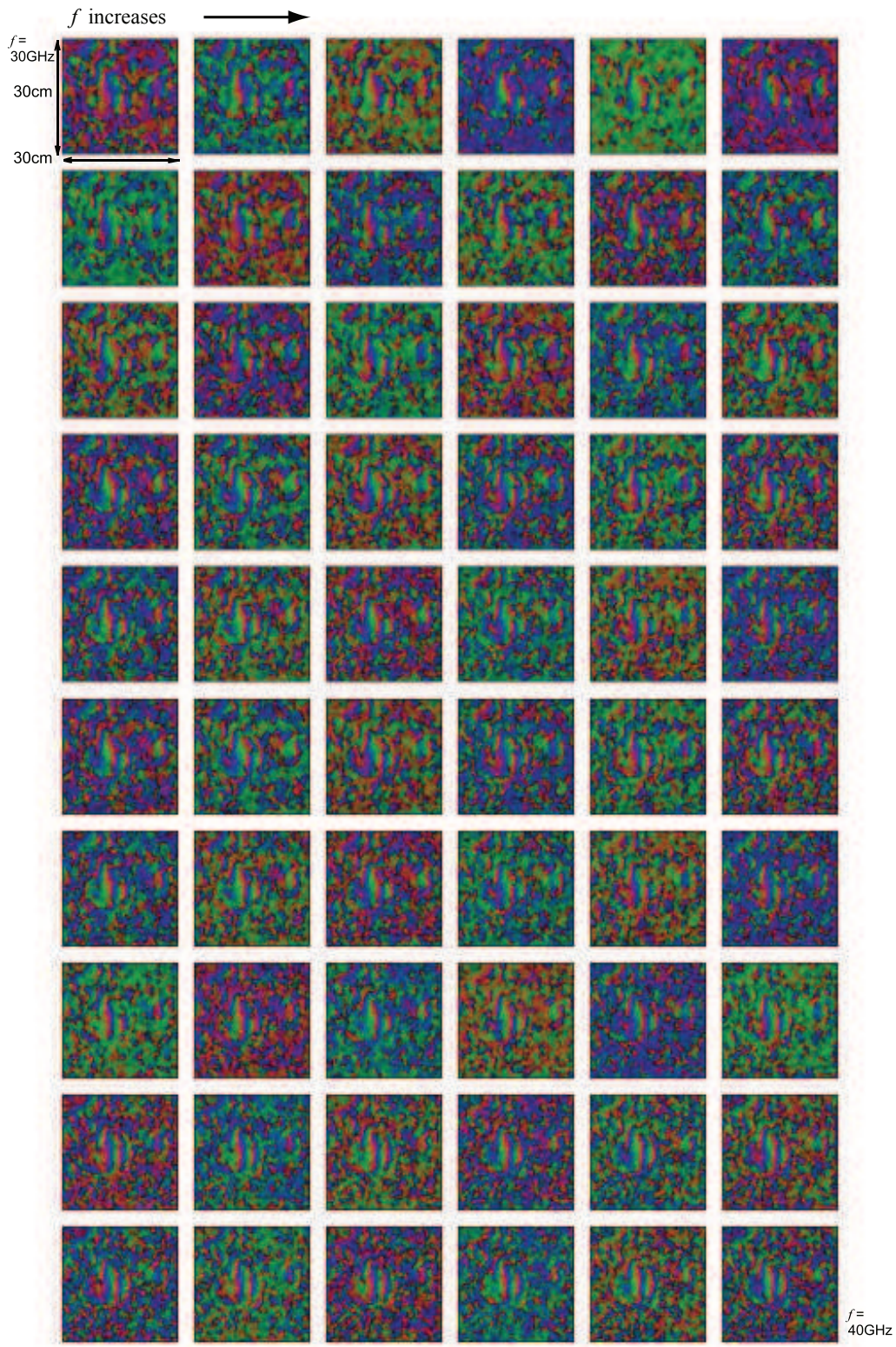

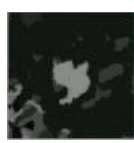

(b)

Fig. 1. (a) raw image showing three dimensional (two dimentional in space $\times$ one dimensional in frequency) complex-amplitude data of scattering / reflection and (b)a result of adaptive segmentation using the CSOM. In (a), the brightness represents the intensity, whereas the hue the phase, and in (b) the grayscale indicates textural class labels (Hara \& Hirose, 2004). 


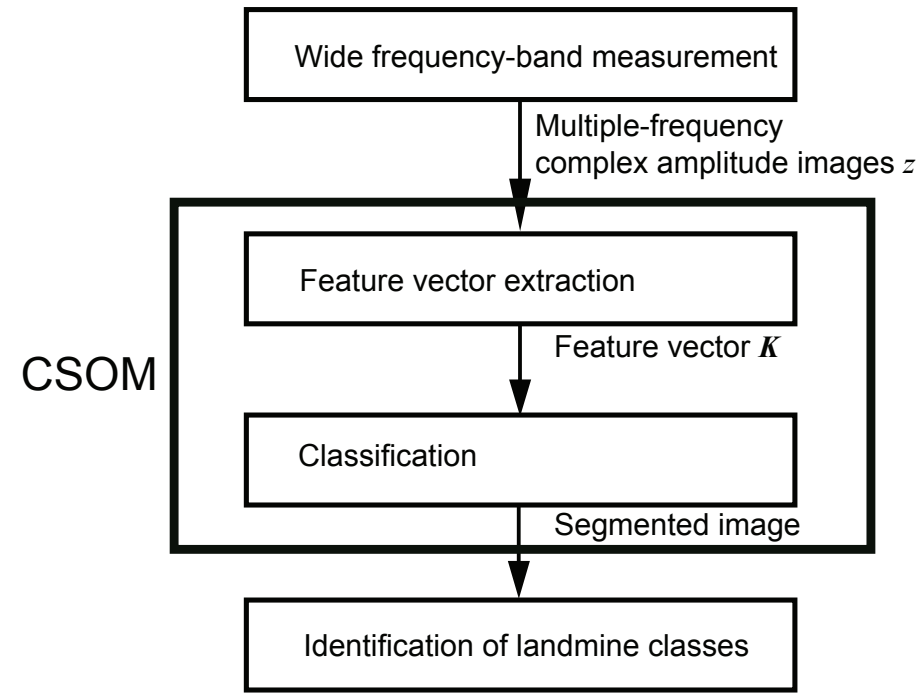

Fig. 2. The overall processing flow (Nakano \& Hirose, 2009b).

yields the phase rotation as well as the amplitude amplification or attenuation. This type of multiplication reduces ineffective degree of freedom in the learning or self-organization to enhance the generalization characteristics in comparison with double-dimensional real-number networks, in spite of the fact that a complex number can be represented as an ordered pair of real numbers. The network dynamics consisting of this elemental rotation and amplification / attenuation leads to significant merits in total, originating from the consistency with the wave-related phenomena and information in the GPRs and other coherent systems (Kawata \& Hirose, 2008) (Tay et al., 2008). For details, see (Hirose, 2006) (Hirose, n.d.).

Among various neurodynamics in the complex domain, the complex-valued self-organizing maps (CSOMs) may possess less features which reflect the complex multiplication, since $\mathrm{SOM}$ in general have two sub-processes in the operation, i.e., winner determination and weight update, both of which sub-processes mostly consist of addition and subtraction in its arithmetics. However, even in such SOM cases, the CSOMs are sensitive to coherence, which is a great advantage in GPRs and other systems that uses coherent waves. In addition, when we employ a complex inner product as the metric to determine the winner, it is found that the advantage is greatly enhanced (Aoyagi et al., 2010).

\section{System construction}

\subsection{Overall construction}

Figure 2 shows the processing flow in our plastic landmine visualization system. We describe the components briefly. First, we operate our system at a stepped-frequency mode to obtain wideband electromagnetic reflection and/or scattering images at multiple frequency points. The details of the system is given in Ref.(Masuyama \& Hirose, 2007). We acquire complex amplitude images at 10 observation frequency points from 8 to $11.6 \mathrm{GHz}$ at an interval of $0.4 \mathrm{GHz}$.

Next, we generate a spatially segmented image by using a CSOM that classifies local texture adaptively. The classification consists of two steps. In the first step, we extract feature vectors 


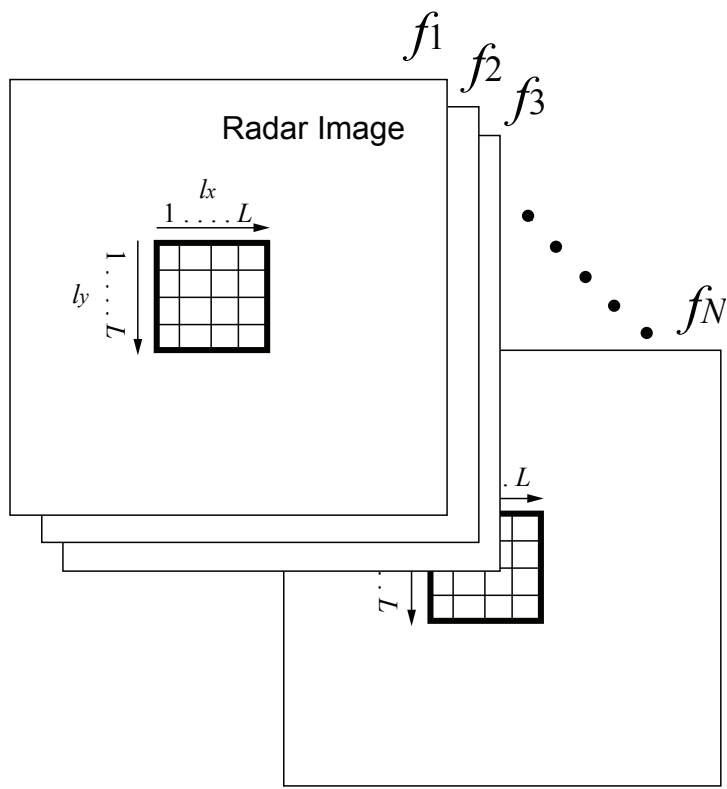

Fig. 3. The scanning window for local textural feature extraction (Nakano \& Hirose, 2009b).

representing local complex-amplitude textural quantity in a local window that sweeps all over the image. As shown in Fig.3, we prepare a sweeping window in each frequency image at a synchronizing real-space location. We calculate correlations between pixel values in the window in terms of real-space relative distance and frequency-domain distance. We assume that the correlation values represent the texture at around the pixel at the window center, and we put the values at the center pixel as the textural feature. In the second step, we classify the extracted feature vectors adaptively by using a CSOM (Hara \& Hirose, 2004). Then we color pixels correspondingly with the resulting classes to generate a segmented spatial image.

Lastly, we identify landmine classes included in the segmented image. We use an associative memory that learns respective features of landmine classes and other objects such as metal fragments, stones and clods. We prepare a set of teacher features for the learning beforehand by observing land pieces where we know the landmine locations.

In this paper, we propose two novel methods in the CSOM processing. One is a dynamics in the feature vector classification, and the other is a feature vector extraction method. The former is utilization of SOM-space topology in the CSOM adaptive classification by introducing a ring CSOM, and the latter is the extraction of local correlation in the frequency domain.

\subsection{Local correlation method to extract textural features}

One of the specific methods in our system is to adopt frequency-domain local correlation in the texture feature extraction. We extract textural features in the spatial and frequency domains as follows. This process corresponds to the human early vision using Gabor filter though we deal with the correlation values directly. In particular, we extend the "local correlation" to the frequency domain in the following manner (Nakano \& Hirose, 2009b). 


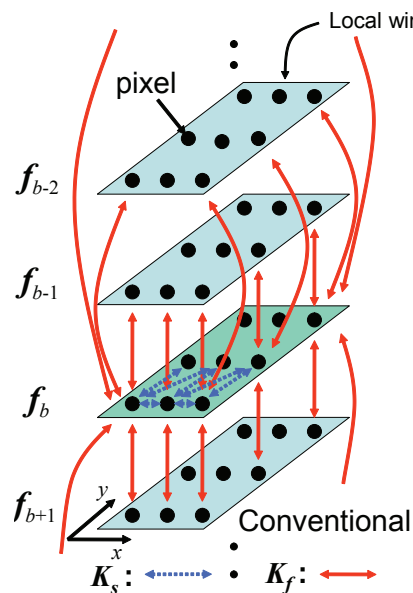

(a)

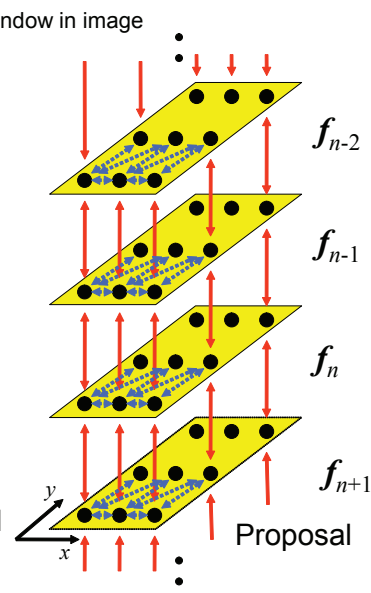

(b)

Fig. 4. (a)Conventional and (b)proposed textural feature extraction methods based on correlation in real-space and frequency domains (Nakano \& Hirose, 2009b).

Figure 4(a) illustrates the conventional method, in which the feature vector $\boldsymbol{K}$ is calculated for complex pixel values $z\left(l_{x}, l_{y}, f\right)$, where the amplitude is represented in decibel $(\mathrm{dB})$, as

$$
\begin{aligned}
\boldsymbol{K} & =\left[M, \boldsymbol{K}_{s}, \boldsymbol{K}_{f}\right] \\
M & =\frac{1}{L^{2}} \sum_{l_{x}=1}^{L} \sum_{l_{y}=1}^{L} z\left(l_{x}, l_{y}, f_{b}\right) \\
\boldsymbol{K}_{s} & =\left[K_{s}(0,0), K_{s}(1,0), K_{s}(0,1), K_{s}(1,1)\right] \\
K_{s}(i, j) & =\frac{1}{L^{2}} \sum_{l_{x}=1}^{L} \sum_{l_{y}=1}^{L} z\left(l_{x}, l_{y}, f_{b}\right) z^{*}\left(l_{x}+i, l_{y}+j, f_{b}\right) \\
K_{f} & =\left[K_{f}\left(f_{1}\right), \cdots, K_{f}\left(f_{N}\right)\right] \\
K_{f}\left(f_{n}\right) & =\frac{1}{L^{2}} \sum_{l_{x}=1}^{L} \sum_{l_{y}=1}^{L} z\left(l_{x}, l_{y}, f_{b}\right) z^{*}\left(l_{x}, l_{y}, f_{n}\right)
\end{aligned}
$$

where $M, \boldsymbol{K}_{s}$, and $\boldsymbol{K}_{f}$ are the mean, real-space-domain correlations, and frequency-domain correlations, respectively. Real-space discrete coordinate $l_{x}$ and $l_{y}$ determine pixel positions in the local window as shown in Fig.3.

We prepare a local observation window of $L \times L$ pixels to extract the local textural feature in the window by calculating correlations between pixel values. In (2)-(6), $M$ is the average of pixel values in the window at a base frequency $f_{b}$, which we select among the 10-frequency observation points in advance. The vector $\boldsymbol{K}_{s}$ in (3) is the local correlations in the $L \times L$ real-space window at $f_{b}$, while $\boldsymbol{K}_{f}$ is the correlations between pixel values at $f_{b}$ and other frequencies $f_{n}$ at identical positions.

The effectiveness of $K_{f}\left(f_{n}\right)$ in (6) as a feature vector was suggested by the following frequency-dependent interference. A plastic landmine usually has parallelism among its 
ceiling, bottom, and air gap inside, if any, which causes interference, whose spectral profile is periodic in the frequency domain. That is to say, we will observe a resonance at integral multiple of a certain frequency periodically in the frequency domain, resulting in a specific peak at certain $f_{n}$ in $K_{f}\left(f_{n}\right)$. We intended to capture this phenomenon in (6). However, we found in our series of experiments that we normally observe only a single peak in the $8-12 \mathrm{GHz}$ band. If we expect multiple frequency peaks, we have to expand the observation bandwidth. However, very high-frequency electromagnetic wave cannot penetrate ground so deep.

Then we have changed our direction. Note that, in the spatial texture case described above, we paid attention to local correlation caused by the Markovian property. In the same way, also in the frequency domain, we decided to calculate the local correlation to observe the frequency space texture in a simple manner.

Figure 4(b) illustrates our proposal, namely the frequency-domain local correlation method, to extract the frequency-domain feature. We define our new $K_{f}$ as

$$
\begin{aligned}
K_{f} & =\left[K_{f}\left(f_{1}\right), \cdots, K_{f}\left(f_{N-1}\right)\right] \\
K_{f}\left(f_{n}\right) & =\frac{1}{L^{2}} \sum_{l_{x}=1}^{L} \sum_{l_{y}=1}^{L} z\left(l_{x}, l_{y}, f_{n}\right) z^{*}\left(l_{x}, l_{y}, f_{n+1}\right)
\end{aligned}
$$

where $\boldsymbol{K}_{f}$ is the feature vector representing the correlation coefficients between the data at adjoining frequency points. This method enables us to eliminate the base frequency $f_{b}$, which means that we do not need to choose a special frequency. To extend this $f_{b}$-free advantage further, we also modify $M$ and $K_{s}(i, j)$ slightly as

$$
\begin{aligned}
M & =\frac{1}{L^{2} N} \sum_{l_{x}=1}^{L} \sum_{l_{y}=1}^{L} \sum_{n=1}^{N} z\left(l_{x}, l_{y}, f_{n}\right) \\
K_{s}(i, j) & =\frac{1}{L^{2} N} \sum_{l_{x}=1}^{L} \sum_{l_{y}=1}^{L} \sum_{n=1}^{N} z\left(l_{x}, l_{y}, f_{n}\right) z^{*}\left(l_{x}+i, l_{y}+j, f_{n}\right)
\end{aligned}
$$

That is, $M$ and $K_{S}$ are averaged for the all used frequency data.

The frequency-domain local correlation method is suitable for the processing in this system. Instead of the radar cross section, we use the texture of complex amplitude when we distinguish plastic landmines and other objects such as metal fragments and stones. If we can obtain a sufficiently high resolution in real-space and frequency domains, we should take into account the correlation between one pixel and another at a certain distance. However, when the system has only a low resolution, it is more effective to concentrate on the correlation between neighboring pixels, in which we can expect useful correlation information.

Additionally, in the proposed method, it is a great merit that we do not need the base frequency $f_{b}$, which was required in the conventional method. Previously we have a number of possible $f_{b}$. As presented below, it is a problem that a different $f_{b}$ results in a different segmentation image. The new method is free from this problem because we have only one way to construct $K$.

\subsection{Utilization of SOM-space topology in the CSOM adaptive classification}

The other method specific to our new system is that we employ a SOM dynamics that utilizes SOM-space topology in the CSOM adaptive classification (Nakano \& Hirose, 2009b). Figure 5 shows the CSOM structure, which forms a ring in the CSOM space. In the CSOM in our 


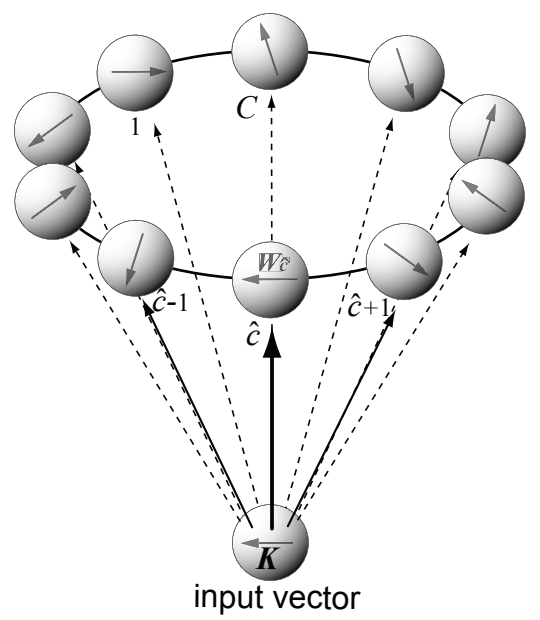

Fig. 5. The ring-CSOM structure. Balls represent reference vectors in the CSOM, and the directions of the arrows show the vector values, among which the winner $\boldsymbol{W}_{\hat{c}}$ and the two neighbors $\boldsymbol{W}_{\hat{c} \pm 1}$ change in the self-organization.

previous system, we classified the feature vectors by using the K-mean clustering algorithm, which is the simplest SOM dynamics, as (Hara \& Hirose, 2004)

$$
\begin{aligned}
\boldsymbol{W}_{\hat{c}}(t+1) & =\boldsymbol{W}_{\hat{c}}(t)+\alpha(t)\left(\boldsymbol{K}-\boldsymbol{W}_{\hat{c}}(t)\right) \\
\alpha(t) & =\alpha(0)\left(1-\frac{t}{T}\right)
\end{aligned}
$$

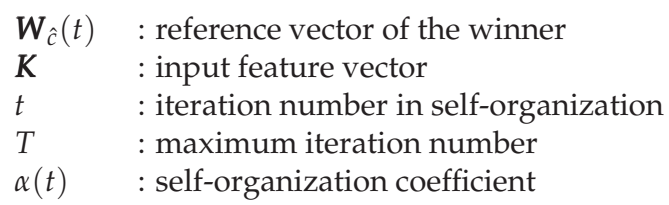

where the winner $W_{\hat{c}}(t)$ is the reference vector nearest to $K$ among all the reference vectors $\boldsymbol{W}_{1}, \boldsymbol{W}_{2}, \ldots, \boldsymbol{W}_{c}, \ldots, \boldsymbol{W}_{C}$. We update $\boldsymbol{W}_{\hat{c}}$ iteratively by presenting $\boldsymbol{K}$ sequentially. In the new system, we also introduce the self-organization of reference vectors at the winner neighbors $(c \pm 1)$ in the SOM space shown in Fig.5 as (Nakano \& Hirose, 2009b)

$$
\begin{aligned}
\boldsymbol{W}_{\hat{c} \pm 1}(t+1) & =\boldsymbol{W}_{\hat{c} \pm 1}(t)+\beta(t)\left(\boldsymbol{K}-\boldsymbol{W}_{\hat{c} \pm 1}(t)\right) \\
\beta(t) & =\beta(0)\left(1-\frac{t}{T}\right)
\end{aligned}
$$

where $\beta(t)$ is another self-organization coefficient for the neighboring classes, which is usually smaller than $\alpha(t)$. The classes $\hat{c} \pm 1$ are neighbors of the winner class $\hat{c}$ in the CSOM space. The CSOM space is used only to determine the winner neighbors, whereas the winner is determined in the $K$ space.

The reason of the modification is as follows. In the previous method, we used the K-means algorithm (11), which is the simplest dynamics in the SOM. Because we have only about 10 


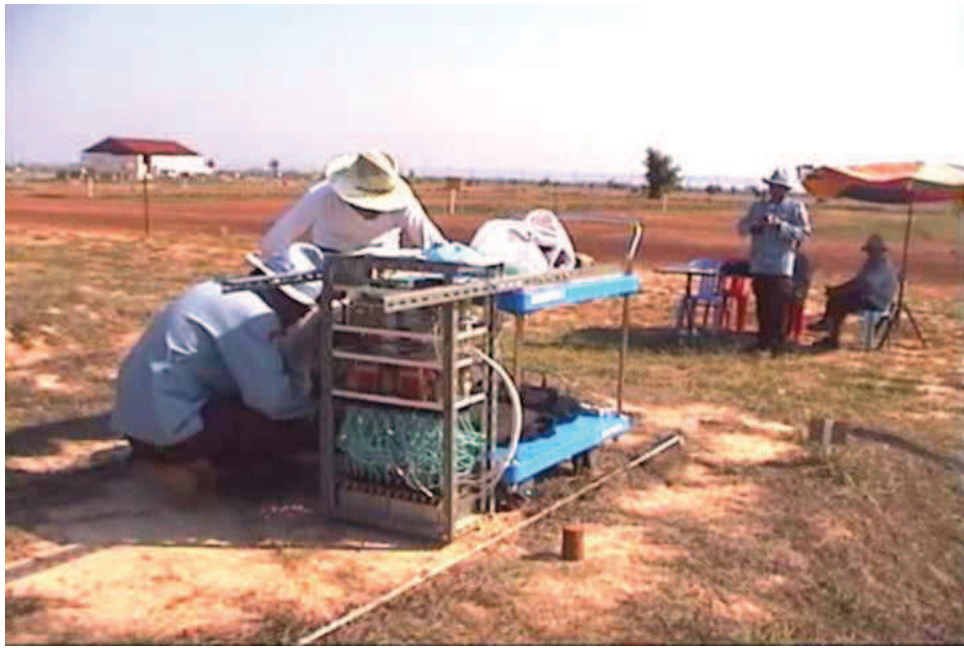

Fig. 6. Photo of our field experiment in Siem Reap, Cambodia (Hirose, 2008).

classes in the adaptive vector quantization in the landmine visualization system, we paid less attention to the SOM-space topology. Nevertheless, we could obtain sufficiently meaningful classification results (Hara \& Hirose, 2004). However, with the present portable visualization system with a lower spatial resolution at the antenna array (Masuyama \& Hirose, 2007), the failure probability in the classification became slightly higher than the first laboratory system. We sometimes fail to extract texture features sufficiently because of the decrease in resolution. As described later, in such an insufficient extraction case, we found that only a small number of classes were used in the vector quantization.

We therefore proposed the utilization of the SOM-space topology in the CSOM adaptive classification, to activate most of the prepared reference vectors, by introducing additional self-organization at neighbor class vectors. In this paper, we change the values of only the two adjacent-class vectors $\boldsymbol{W}_{c \pm 1}$ as shown in (13). The neighbor vector number is small because the number of the totally prepared classes is small, i.e., only about 10 . The structure of the CSOM should also be suitable for the small size, namely, one-dimensional as shown in Fig.5.

\section{Experiments and results}

Figure 6 shows the field experiment conducted in Cambodia to evaluate our system for laterite soil which has a high permeability and hence a larger clutter. At the site, we also have frequent heavy rainfall, resulting in temporary higher permittivity of the soil lasting for several hours to a few days. Table 1 shows the parameters used in the following experiments. We have determined the values of $\alpha(0)$ and $\beta(0)$ empirically. We bury a mock plastic landmine under ground iteratively. We change the burial situation every time, including the ground surface and underground. The surface-roughness amplitude is about $2 \mathrm{~cm}$ peak-to-peak. In any case, the landmine is buried at around the center of the observation area.

Figure 7(a) shows an experimental result (Result 1) obtained for lightly wet laterite soil. The numbers show the observation frequencies. The upper blue maps show the amplitude data, while the lower color maps show the phase data. Scales of amplitude and phase are shown at the top. The position in every map corresponds to the position in real space. As mentioned 


\begin{tabular}{|r|l|}
\hline Target (Plastic landmine) \\
\hline Size & $78 \mathrm{~mm}^{\phi}, 40 \mathrm{~mm}$ high \\
Burial depth & $2 \sim 3 \mathrm{~cm}$ \\
\hline \multicolumn{2}{|c|}{ System } \\
\hline Antenna height & $2 \sim 3 \mathrm{~cm}$ above ground \\
Window size & $L=4$ \\
Frequency number & $N=10$ \\
Class number & $C=10$ \\
Initial learning coefficients & $\alpha(0)=0.4$ \\
& $\beta(0)=0.1$ \\
Maximum learning iteration & $T=10$ \\
\hline
\end{tabular}

Table 1. Parameters of target and system (Nakano \& Hirose, 2009b).

above, we use these complex amplitude data obtained at the 10 frequency points. Figure 7 (b) shows segmented images generated with the previous method. The numbers are base frequencies $f_{b}$ used respectively. We can choose feature vectors $\boldsymbol{K}$ in 10 ways because there are 10 possible $f_{b}$. Each gray level indicates one of the 10 classes. We can find a segmented area at the buried plastic landmine position at $f_{b}=8 \mathrm{GHz}$ and $8.8 \mathrm{GHz}$. However, we cannot at other $f_{b}$. Figure 7 (c) shows the result of segmentation by utilizing the SOM-space topology in the $\mathrm{CSOM}$ adaptive classification. We find that there are more classes used in the classification, i.e., 10 classes in most cases, than that in the case of the previous method. We can confirm that we can classify the landmine area appropriately at most $f_{b}$. For example, also at $8.4 \mathrm{GHz}$ and $9.2 \mathrm{GHz}$, we are successful in the segmentation this time. These results reveal that we can improve the performance of classification by the utilization of the SOM-space topology in the CSOM. Figure 7 (d) shows the segmentation result obtained by the frequency-domain local correlation method as well as the utilization of SOM-space topology. As mentioned before, there is only one manner to extract feature vectors $K$ in this proposed method because we have no $f_{b}$. Here we show four result examples for various initial reference vectors in the CSOM since the result of the CSOM may depend on the initial reference-vector values. In all the cases, the landmine area is segmented correctly. We confirm a high robustness of the present method with the two proposal.

Figure 8(a) shows a measurement result (Result 2) obtained for highly wet laterite soil just after a heavy rain, which means a more difficult situation than that of Fig.7(a). The landmine classification seems more difficult in this case than that of Fig.7 because the calibration of direct coupling components (Masuyama et al., 2007) is somewhat sensitive to noise, occasionally resulting in insufficient compensation of antenna-selection-mode dependent amplitude. Figure 8(b) shows the segmented images obtained with the previous method. We can classify the landmine area only when $f_{b}=9.2 \mathrm{GHz}$. We completely failed in the segmentation at other $f_{b}$. Figure 8 (c) shows a result by utilizing the SOM-space topology in the CSOM. We can segment the landmine area only at $9.2 \mathrm{GHz}$ again. Figure 8 (d) shows the results obtained by employing the two proposed methods. It is confirmed that we can classify the landmine area perfectly. We show four results for various initialization again. The results indicate that we can segment landmine areas stably. In addition, we recognize that more classes are used for the classification in Fig.8(d) than in Fig.8(c) despite we use the same dynamics for the classification in the CSOM. For this reason, we can extract more characteristic feature quantities with the frequency-domain local correlation method than that with the previous one. 

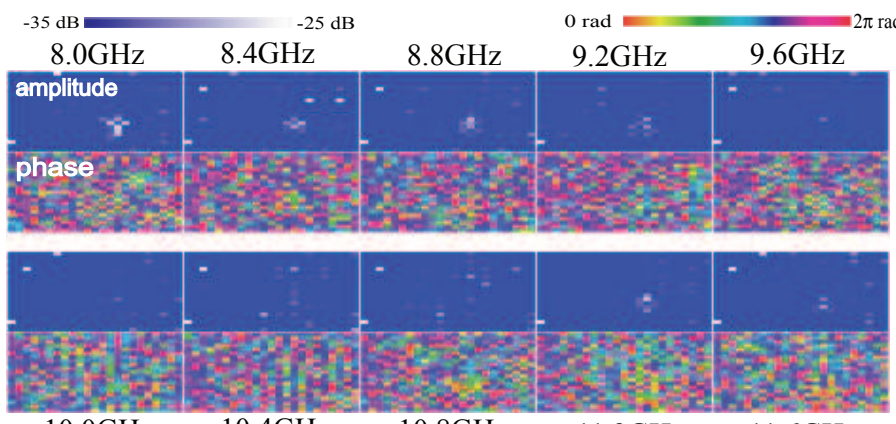

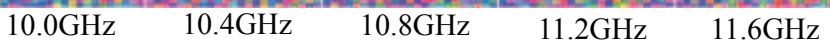

(a)

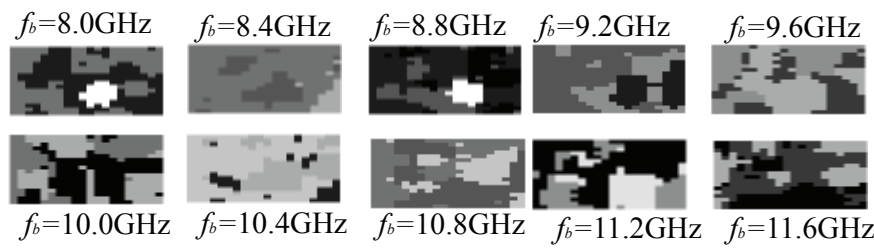

(b)

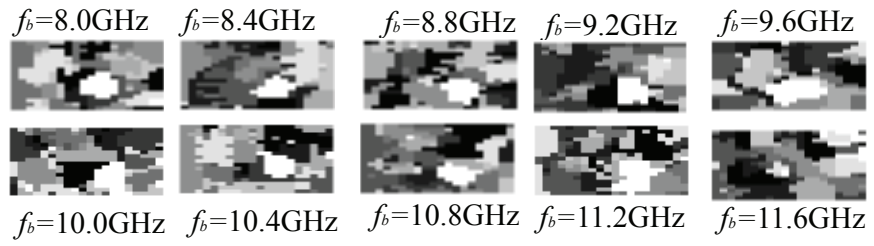

(c)
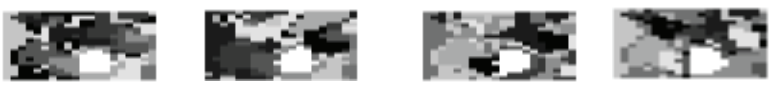

(d)

Fig. 7. Experimental results 1 for lightly wet laterite soil. (a)Amplitude and phase images at 10 frequency points, (b)classification results with the previous method. Numbers denote base frequency $f_{b}$, (c)classification results with utilization of SOM-space topology in the CSOM. Numbers denote base frequency. (d)Classification results with utilization of SOM-space topology in the CSOM and frequency-domain local correlation method. The four images show the results with various initial reference vectors in the CSOM (Nakano \& Hirose, 2009b).

\section{Summary}

We reviewed recently proposed two techniques, used in stepped-frequency GPRs, to improve the performance of the GPR system to visualize plastic landmines. One is to employ local correlation as the feature vector components in the frequency domain as well as spatial domain. It extracts complex texture information better and, at the same time, eliminates the need of the base frequency, which had to be chosen by the user as one of the tuning parameters 

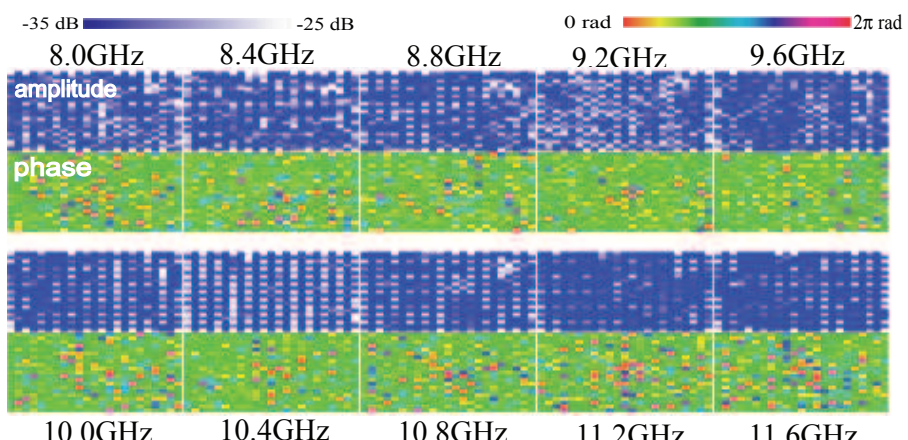

(a)

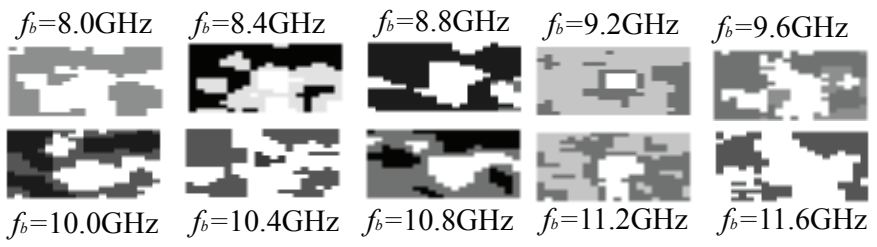

(b)

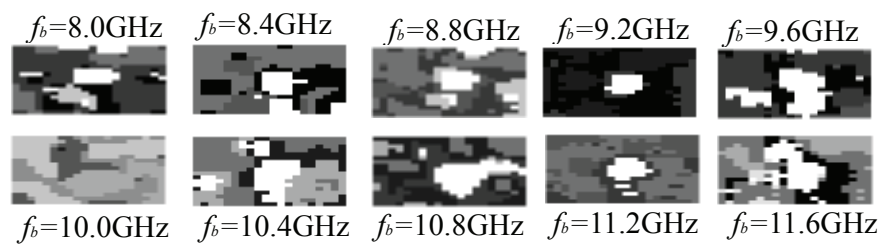

(c)
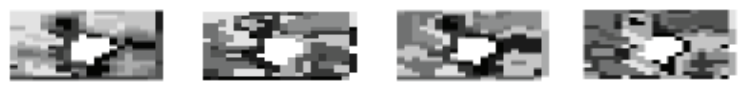

(d)

Fig. 8. Experimental results 2 for heavily wet laterite soil. (a)Amplitude and phase images at 10 frequency points, (b)classification results with the previous method. Numbers denote base frequency $f_{b}$, (c)classification results with utilization of SOM-space topology in the CSOM. Numbers denote base frequency. (d)Classification results with utilization of SOM-space topology in the CSOM and frequency-domain local correlation method. The four images show the results with various initial reference vectors in the CSOM (Nakano \& Hirose, 2009b).

in the previous system. The other technique is to utilize SOM-space topology in the CSOM adaptive classification to stabilize the classification process. Unlike the K-means algorithm, we can use most of the prepared classes in the learning vector quantization. We presented the experimental results obtained in Cambodia to demonstrate better and stable visualization of the plastic landmine. 


\section{References}

Aizenberg, I. N., Aizenberg, N. N. \& Joos, V. (2000). Multi-Valued and Universal Binary Neurons - Theory, Learning and Applications -, Kluwer Academic Publishers, Boston / Dordrecht / London.

Aoyagi, T., Radenamad, D., Nakano, Y. \& Hirose, A. (2010). Complex-valued self-organizing map clustering using complex inner product in active mmillimeter-wave imaging, Proceedings of the International Joint Conference on Neural Networks (IJCNN) 2010 Barcelona, IEEE/INNS, Barcelona, pp. 1346-1351.

Bourgeois, J. M. \& Smith, G. S. (1998). A complete electromagnetic simulation of the separated-aperture sensor for detecting buried land mines, IEEE Transactions on Antennas and Propagation 46(10): 1419-1426.

Hara, T. \& Hirose, A. (2004). Plastic mine detecting system using complex-valued self-organizing map that deals with multiple-frequency interferometric images, Neural Networks 17(8-9): 1201-1210.

Hara, T. \& Hirose, A. (2005). Adaptive plastic-landmine visualizing radar system: effects of aperture synthesis and feature-vector dimension reduction, IEICE Transactions on Electronics E88-C(12): 2282-2288.

Hirose, A. (2006). Complex-Valued Neural Networks, Springer-Verlag, Heidelberg, Berline, New York.

Hirose, A. (2008). An adaptive ground penetrating radar imaging system based on complex-valued self-organizing map - recent progress and experiments in cambodia -, Proceedings of the World Congress on Computational Intelligence (WCCI) 2008 Hong Kong - International Joint Conference on Neural Networks (IJCNN) 2008 Hong Kong -, pp. $1610-1615$.

Hirose, A. (2009). Complex-valued neural networks: The merits and their origins, Proceedings of the International Joint Conference on Neural Networks (IJCNN) 2009 Atlanta, IEEE INNS, pp. 1237-1244.

Hirose, A. (n.d.). Complex-valued neural networks (Tutorial, International Joint Conference on Neural Networks (IJCNN) 2009 Atlanta) (IEEE Computational Intelligence Society (CIS) video archive.

URL: http://ewh.ieee.org/cmte/cis/mtsc/ieeecis/video_tutorials.htm

Hirose, A. (ed.) (2003). Complex-Valued Neural Networks: Theories and Applications, World Scientific Publishing Co. Pte. Ltd.

Hirose, A., Toh Jiayun, A. \& Hara, T. (2005). Plastic landmine identification by multistage association, IEICE Tech. Rep. (NC2004-156).

Kawata, S. \& Hirose, A. (2008). Frequency-multiplexing ability of complex-valued Hebbian learning in logic gates, International Journal of Neural Systems 12(1): 43-51.

Kobayashi, T., Feng, X. \& Sato, M. (2004). A gpr for landmine detection using an array antenna, International Symposium on Antennas and Propagation (ISAP) Sendai.

Mandic, D. P. \& Goh, V. S. L. (2009). Complex Valued Nonlinear Adaptive Filters - Noncircularity, Widely Linear and Neural Models, Wiley.

Masuyama, S. \& Hirose, A. (2007). Walled LTSA array for rapid, high spatial resolution, and phase sensitive imaging to visualize plastic landmines, IEEE Transactions on Geoscience and Remote Sensing 45(8): 2536-2543.

Masuyama, S., Yasuda, K. \& Hirose, A. (2007). Removal of direct coupling in a walled-LTSA array for visualizing plastic landmines, International Symposium on Antennas and Propagation (ISAP) 2007 Niigata, pp. 1238-1241. 
Masuyama, S., Yasuda, K. \& Hirose, A. (2008). Multiple mode selection of walled-ltsa array elements for high resolution imaging to visualize antipersonnel plastic landmines, IEEE Geoscience and Remote Sensing Letters 5(4): 745-749.

Montoya, T. P. \& Smith, G. S. (1999). Land mine detection using a ground-penetrating radar based on resistively loaded vee dipoles, IEEE Trans. Antenna and Propagation 47(12): 1795-1806.

Moriyama, T., Nakamura, M., Yamaguchi, Y. \& Yamada, H. (1997). Radar polarimetry applied to the classification of target buried in the underground, Wideband Interferometric Sensing and Imaging Polarimetry, Vol. 3210 of Proc. of SPIE, pp. 182-189.

Nakano, Y. \& Hirose, A. (2009a). Estimation of landmine class likelihood by evaluating the degree of matching of features in ring-CSOM space, Technical report, IEICE Technical Report (NC2008-169).

Nakano, Y. \& Hirose, A. (2009b). Improvement of plastic landmine visualization performance by use of ring-CSOM and frequency-domain local correlation, IEICE Transactions on Electronics E92-C(1): 102-108.

Nakano, Y. \& Hirose, A. (2009c). Radar Technology, In-Tech, chapter Adaptive ground penetrating radar systems to visualize antipersonnel plastic landmines based on local texture in scattering and reflection data in space and frequency domains, pp. 127-140.

Nitta, T. (ed.) (2009). Complex-Valued Neural Networks: Utilizing High-Dimensional Parameters, IGI-Global.

Peters Jr., L. \& Daniels, J. J. (1994). Ground penetrationg radar as a surface environmental sensing tool, Proceedings of the IEEE 82,No.12: 1802-1822.

Sato, M., Fujiwara, J., Fenga, X. \& Kobayashi, T. (2005). Dual sensor ALIS evaluation test in Afghanistan, IEEE Geoscience and Remote Sensing Society Newsletter pp. 22-27.

Sato, M., Hamada, Y., Feng, X., Kong, F.-N., Zeng, Z. \& Fang, G. (2004). GPR using an array antenna for landmine detection, Near Subsurface Geophysics 2: 7-13.

Sato, M., Takahashi, K., Feng, X. \& Kobayashi, T. (2006). Stepped-frequency handheld demining dual sensor alis, Proceeding of 11th International Conference on Ground Penetrating Radar, p. UXO.10.

Sato, M. \& Takeshita, M. (2000). Estimation of subsurface fracture roughness by polarimetric borehole radar, IEICE Transactions on Electronics E83-C(12): 1881-1888.

Shrestha, S. M., Arai, I. \& Tomizawa, Y. (2004). Landmine detection using impulse ground penetrating radar, Internationall Symposium on Antennas and Propagation (ISAP) Sendai.

Tay, C. S., Tanizawa, K. \& Hirose, A. (2008). Error reduction in holographic movies using a hybrid learning method in coherent neural networks, Applied Optics 47(28): 5221-5228.

Widrow, B., McCool, J. \& Ball, M. (1975). The complex lms algorithm, Proceedings of the IEEE 63: 719-720. 


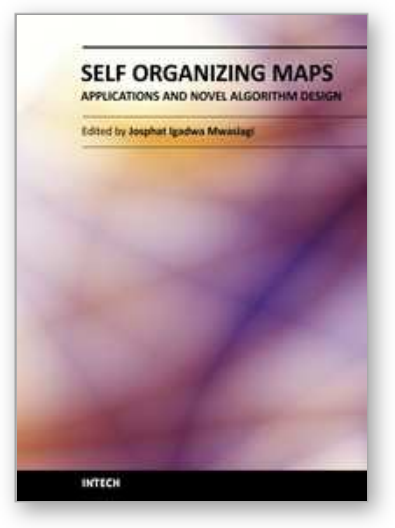

\author{
Self Organizing Maps - Applications and Novel Algorithm Design \\ Edited by Dr Josphat Igadwa Mwasiagi
}

ISBN 978-953-307-546-4

Hard cover, 702 pages

Publisher InTech

Published online 21, January, 2011

Published in print edition January, 2011

Kohonen Self Organizing Maps (SOM) has found application in practical all fields, especially those which tend to handle high dimensional data. SOM can be used for the clustering of genes in the medical field, the study of multi-media and web based contents and in the transportation industry, just to name a few. Apart from the aforementioned areas this book also covers the study of complex data found in meteorological and remotely sensed images acquired using satellite sensing. Data management and envelopment analysis has also been covered. The application of SOM in mechanical and manufacturing engineering forms another important area of this book. The final section of this book, addresses the design and application of novel variants of SOM algorithms.

\title{
How to reference
}

In order to correctly reference this scholarly work, feel free to copy and paste the following:

Akira Hirose and Yukimasa Nakano (2011). Applications of Complex-Valued Self-Organizing Maps to Ground Penetrating Radar Imaging Systems, Self Organizing Maps - Applications and Novel Algorithm Design, Dr Josphat Igadwa Mwasiagi (Ed.), ISBN: 978-953-307-546-4, InTech, Available from:

http://www.intechopen.com/books/self-organizing-maps-applications-and-novel-algorithm-design/applicationsof-complex-valued-self-organizing-maps-to-ground-penetrating-radar-imaging-systems

\section{INTECH}

open science | open minds

\author{
InTech Europe \\ University Campus STeP Ri \\ Slavka Krautzeka 83/A \\ 51000 Rijeka, Croatia \\ Phone: +385 (51) 770447 \\ Fax: +385 (51) 686166 \\ www.intechopen.com
}

\author{
InTech China \\ Unit 405, Office Block, Hotel Equatorial Shanghai \\ No.65, Yan An Road (West), Shanghai, 200040, China \\ 中国上海市延安西路65号上海国际贵都大饭店办公楼 405 单元 \\ Phone: +86-21-62489820 \\ Fax: +86-21-62489821
}


(C) 2011 The Author(s). Licensee IntechOpen. This chapter is distributed under the terms of the Creative Commons Attribution-NonCommercialShareAlike-3.0 License, which permits use, distribution and reproduction for non-commercial purposes, provided the original is properly cited and derivative works building on this content are distributed under the same license. 